\title{
Effectiveness of the Passive Lingual Arch for E Space Maintenance in Subjects with Anterior or Posterior Rotation of the Mandible: A Retrospective Study
}

\author{
Grazia Fichera Mariagrazia Greco Rosalia Leonardi \\ Department of Orthodontics, University of Catania, Catania, Italy
}

Key Words

Molar drift • Mandible growth patterns $\cdot$ Arch length

\begin{abstract}
Objective: The aim of this study was to determine whether or not lingual arches in subjects with anterior or posterior mandible rotation can produce different effects in the prevention of first permanent molar mesial migration. Subjects and Methods: Forty-two patients with a mean age of $9 \pm 0.8$ years were selected from the Department of Orthodontics, University of Catania, Italy. These subjects were treated with a passive mandibular fixed lingual arch. The sample was divided into 3 groups according to the gonial angle in order to establish the pattern of mandible growth: mandibular posterior rotation (MPR), mandibular growth in straight-downward direction (MSD) and mandibular anterior rotation (MAR). Lateral cephalograms and study models of the patients before and after treatment were examined to determine any positional changes of the mandibular first molars and incisors. Results: Statistically significant differences between the MPR group and the other 2 (MSD and MAR) were found as regards mandibular first molar and incisor positional changes. No significant differences were found between the MSD and MAR groups. Conclusion: The results indicate that the effect of lingual arch is influenced by mandibular growth patterns. In patients with MPR, the lingual arch preserves arch length but the mesial migration of first permanent molars is not completely blocked.

Copyright $\odot 2011$ S. Karger AG, Basel
\end{abstract}

\section{Introduction}

In orthodontics, the mandibular fixed lingual arch, used as a passive appliance, is a commonly accepted procedure. As such, it has been used to preserve anchorage in the lower arch, maintain arch length by controlling the anterior movement of the molars, and prevent the mandibular incisors from collapsing in a lingual direction. Moreover, it is used to prevent the anchor molars from tipping forward into the space created by the extraction of second primary molars [1,2], especially when primary cuspids have also been extracted.

According to Moyers et al. [3], as much as $4.8 \mathrm{~mm}$ of space can become available as the permanent canines and premolars replace their primary successors. Generally speaking, the first molars move mesially into the 'leeway' or ' $E$ ' space during the transition from mixed to permanent dentition; this, in turn, leads to a decrease in arch length [4]. The lingual arch maintains the arch perimeter by preventing mesial tipping or mandibular first molar drift. The molar positions are stabilized, to some extent, against the mandibular incisor thanks to the appliance, which also prevents the incisor from tipping lingually $[5,6]$.

Several studies have shown the effectiveness of lingual arches as space holders [7-10]. However, this type of effectiveness was also reported as having a wide range of variability as stated by Brennan and Gianelly [11], ranging from an average decrease of $-0.44 \pm 1.35 \mathrm{~mm}$ to a gain of $+2.9 \mathrm{~mm}$. They hypothesized that the different magnitude

\section{KARGER}

Fax +4161306 1234 E-Mail karger@karger.ch www.karger.com

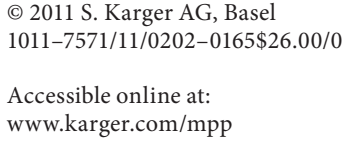

Rosalia Leonardi

Department of Orthodontics, University of Catania

via S. Sofia 78

IT-95128 Catania (Italy)

Tel./Fax +390953 782 645, E-Mail rleonard@ unict.it 
of space preservation was due to changes in the incisor and molar positions which had previously been influenced by facial growth. Hence, it has been agreed that there are differences in arch length development in subjects with anterior or posterior mandible rotation $[12,13]$. In fact, a mandibular anterior rotation (MAR) usually results in an increase in arch length, while posterior rotation decreases it. Accordingly, Ricketts [14] observed that the mesial drift of the first lower molar was significantly greater in subjects with dolichofacial growth patterns compared to those with a brachyfacial growth pattern.

Yet, despite its widespread use in orthodontics, some relevant clinical questions remain to be addressed, and among these is the effective relationship between lingual arch and mandible growth patterns. Therefore, the purpose of this study was to test the 'null hypothesis' that lingual arches produce no significantly different effect in preventing the mesial migration of first permanent molars and in maintaining E space during the development of the late mixed and early permanent dentition in subjects with anterior or posterior mandible rotation.

\section{Subjects and Methods}

\section{Sample}

A retrospective study was conducted with 42 Caucasian patients (20 males and 22 females); the mean age at the beginning of the treatment was $9 \pm 0.8$ years, and at the end of treatment $12 \pm 0.6$ years. At the Department of Orthodontics, University of Catania, Italy, these subjects were treated with a passive mandibular fixed lingual arch, which was used solely as a means of saving E space [11]. The patients had to meet the following inclusion criteria: patients were in early transitional dentition with the 4 lower incisors erupted; pre- (T0) and posttreatment (T1) lateral cephalometric radiographs of good quality; pre- (T0) and posttreatment (T1) study models of good quality, and subjects treated solely with a mandibular fixed lingual arch on the permanent first molars. The following exclusion criteria were used: complex craniofacial deformities or syndromes; mechanical obstacle to the eruption, such as tumors, odontomas or cysts and congenitally or prematurely missing teeth.

The control group was composed of 18 patients ( 8 males and 10 females) with a mean age at the beginning of the observation period of $9.2 \pm 0.6$ years and, at the end of this period, $11.8 \pm 0.6$ years. They were selected, by the same criteria as the treatment group, from patients attending the Orthodontic Clinic of Catania University, Italy, who had received no immediate orthodontic therapy. All patients were examined at a 3-week interval.

Informed consent was obtained, for both the study and control groups, from the patients' parents. This study was approved by the Regional Committee for Medical Research Ethics.

The sample of subjects treated with lingual arch was divided into 3 subgroups, according to the gonial angle values [15] (fig. 1): (1) the mandibular posterior rotation (MPR) group - 18 subjects

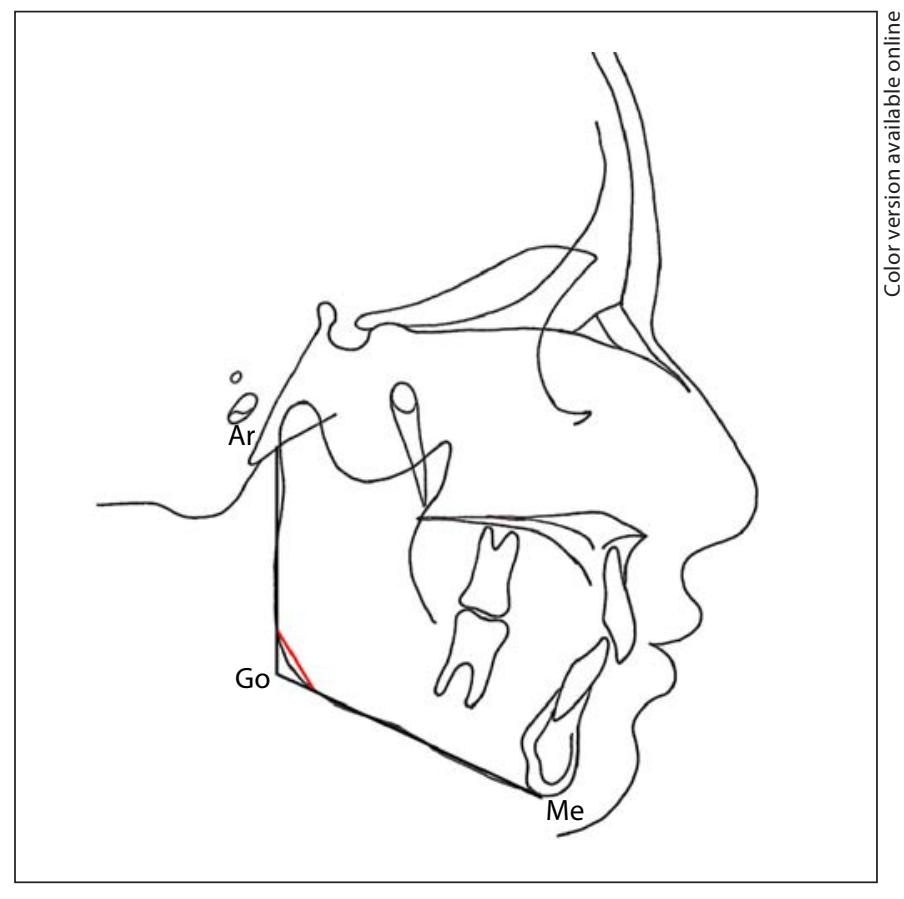

Fig. 1. Linear measurements to calculate gonial angle: Ar-Go (ramus height); Go-Me (mandibular corpus). Ar = Articulare (the point of intersection of the dorsal contour of the condylar head and the contour of the external cranial base); Go = gonion (the midpoint of the mandibular angle between the mandibular ramus and corpus); $\mathrm{Me}=$ menton (the extreme inferior point of the mandibular symphysis).

with mandibular growth in a clockwise direction (articulare-gonion-menton, Ar-Go-Me $\geq 136^{\circ}$ ); (2) the mandibular growth in straight-downward direction (MSD) group - 12 subjects (Ar-Go$\mathrm{Me}=130 \pm 6^{\circ}$ ), and the MAR group - 12 subjects with mandibular growth in a counterclockwise direction (Ar-Go-Me $\leq 124^{\circ}$ ).

The lingual arches used in this study were constructed of 0.036 -inch stainless steel rounded wire. The wires were formed on stone casts and were constructed to lie as low as possible in the cingulum area of the incisors. In addition, the wires were shaped in such a way as to contact as many of the mandibular teeth as was feasible without exerting force on any area, thus making them completely passive. The wire was made to contact the cingulum region of the incisors and was soldered to the lingual surfaces of the first molar bands. The patients were treated and observed by the same operator (G.F.). The patients were required to wear the lingual arch constantly until canines and premolars erupted.

\section{Radiographic and Cephalometric Analysis}

Both pre- and posttreatment lateral cephalograms were gathered for all 60 subjects. The lateral cephalograms were manually traced by one investigator (G.F.) and verified for anatomic contour landmark location and tracing superimposition by another (R.L.). Any disagreements were resolved by retracing the landmark, or structure, to the mutual satisfaction of both investigators. Lateral cephalograms were traced by using acetate tracing 




Fig. 2. Portions of mandible used for superimposition (Björk method). Points identified for measurements: 6 mesiobuccal cusp of the mandibular first molars, 1 incisal edge of the mandibular central incisors, the centers of resistance $(\mathrm{Cr})$ of the incisor $(1 \mathrm{Cr})$ and the molar $(6 \mathrm{Cr})$.

paper placed on a standard light box in a darkened room with a Pentel 0.5-mm lead pencil.

In the lateral cephalograms of the subjects treated with lingual arch, the gonial angle was evaluated to establish the pattern of mandible growth, thus the sample was divided into subgroups. The gonial angle is an angle formed by the intersection of a line traced between Ar and Go (ramus height) and a line traced between Go and Me (mandibular corpus) (fig. 1). This measurement is calculated according to the Jarabak cephalometric analysis, which tells us whether we have a square or an obtuse mandible growth. The tracings of the mandible in each case were superimposed using Björk's method [12, 13] (fig. 2) with the following landmarks: cortical outline of the mandibular canal; lower border of the molar tooth germ; inner cortical border of the inferior portion of the symphysis; most anterior segment of the symphysis; and trabecular patterns in the lower portion of the symphysis.

Changes in tooth position were measured at the incisal edge of the mandibular central incisors, at the mesiobuccal cusp of the mandibular first molars, at the centers of resistance $(\mathrm{Cr})$ of the incisor and the molar, and at the long axis of the incisor and molar. The incisal edge, the mesiobuccal cusp, the $\mathrm{Cr}$ of the incisor and the molar and the long axis of the incisor and the molar were identified on the pretreatment films and transferred to the posttreatment films by superimposing a tooth on itself. The $\mathrm{Cr}$ was located arbitrarily at the bifurcation of molar roots and at a point a third of the root length apical of the alveolus in the incisor teeth (fig. 2). The long axis of the mandibular first molar was drawn from the mesiobuccal cusp to the mesial root tip; for the mandibular incisor the long axis was drawn from the incisal edge to the root apex.

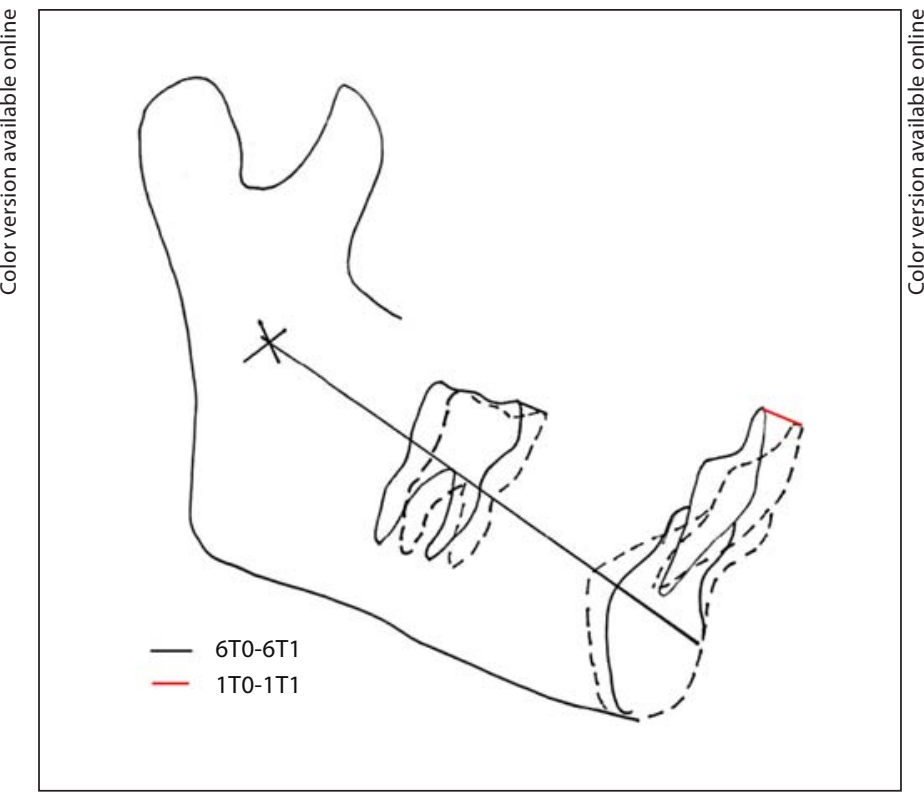

Fig. 3. Positional change of mesiobuccal cusp of the mandibular first molars and incisal edge of the mandibular central incisors.

The following variables were studied: (1) distance from the position of the mesiobuccal cusp of the mandibular first molar at T0 to that at T1 (6T0-6T1) (fig. 3); (2) distance from the position of the $\mathrm{Cr}$ of the mandibular first molar at T0 to that at T1 $[6(\mathrm{Cr})$ T0-6(Cr)T1]; (3) angle formed by the intersection of the long axis of the mandibular first molar at T0 and that at T1 (6 angular); (4) distance from the position of the mandibular central incisor at T0 to that at T1 (1T0-1T1) (fig. 3); (5) distance from the position of the $\mathrm{Cr}$ of the mandibular central incisor at $\mathrm{T} 0$ to that at $\mathrm{T} 1$ $[1(\mathrm{Cr}) \mathrm{T} 0-1(\mathrm{Cr}) \mathrm{T} 1]$; and (6) angle formed by the intersection of the long axis of the mandibular central incisor at T0 and the long axis of the mandibular central incisor at T1 (1 angular).

\section{Study Model Analysis}

The pre- and posttreatment study models were gathered for all 60 subjects. Intermolar width, intercanine width and arch length were measured in millimeters on study cast records of the patients at $\mathrm{T} 0$ and $\mathrm{T} 1$. The intermolar width was the distance between the central fossae of the left and right permanent first molars. The intercanine width was the distance between the canine cusp tips or estimated cusp tips if wear facets were present. Arch length was the combined distance from the mesial contact points of the permanent first molars to the contact point between the permanent central incisors, on both the left and the right sides of the arch. When a difference existed between the 2 sides of a cast, the averages were calculated [10]. All measurements were taken with malleable brass wire and then calculated by digital callipers and recorded to the nearest $0.02 \mathrm{~mm}$. 


\section{Statistical Analysis}

The arithmetic mean and standard deviation were calculated for each variable. Measurements regarding changes in molar and incisor positions, intermolar width, intercanine width and arch length were compared between the 2 groups (treatment and control). The two-tailed t test for paired data was used to test the null hypothesis that there were no differences in the changes between the 2 groups. The null hypothesis would be rejected at a significance level of $<0.05$. The T0-T1 changes in molar and incisor positions, intermolar width, intercanine width and arch length in the 3 groups (MPR, MSD and MAR) were compared by means of the KruskalWallis test with Bonferroni correction for multiple comparisons $(p<0.05)$. All statistical analyses were performed using the Statistical Package for the Social Sciences personal computer version (SPSS for Windows, release 16.0; SPSS Inc., Chicago, Ill., USA).

\section{Measurement Reliability}

For the evaluation of the interoperator error, the complete measurement procedure was repeated by the operator on 10 randomly selected cephalograms and study models after an interval of 12 days. The percentage error was calculated using Dahlberg's formula (mean square error $\mathrm{S} 2=\Sigma \mathrm{d} 2 / 2 \mathrm{n} ; \mathrm{d}=$ difference between repeated measurements, $\mathrm{n}=$ number of radiographs recorded). The errors ranged from 0.3 to $0.6 \mathrm{~mm}$ for linear measurements, and from 0.2 to $0.4^{\circ}$ for angular measurements.

\section{Results}

The difference between the mean changes seen in the treatment and the control (untreated) groups is summarized in table 1. Measurements for the treatment group reflect a $0.6-\mathrm{mm}$ minimal mesial drift of the mesiobuccal cusp, the molar $\mathrm{Cr}$ moved forward by $0.3 \mathrm{~mm}$ and the molar tipped backward by $-0.06^{\circ}$. In the treatment group, the incisal edge advanced by $0.5 \mathrm{~mm}$, the incisor $\mathrm{Cr}$ moved forward by $0.3 \mathrm{~mm}$ and the incisor tipped forward by $0.7^{\circ}$. In the control group there was a $1.9-\mathrm{mm}$ mesial drift of the mesiobuccal cusp, the molar $\mathrm{Cr}$ came forward by $1.3 \mathrm{~mm}$ and the molar tipped forward by $1.9^{\circ}$; the incisal edge moved back by $0.4 \mathrm{~mm}$, the incisor Cr moved back by $-0.2 \mathrm{~mm}$ and the incisor tipped backward by $-2.1^{\circ}$. All the differences between the treatment group and the control groups were statistically significant $(\mathrm{p}<$ 0.05).

The mean changes and standard deviations in the treatment and control (untreated) groups, measured in the study models, are shown in table 2 . All the differences between the treatment and control groups were statistically significant $(\mathrm{p}<0.05)$. A $1.8-\mathrm{mm}$ decrease in arch length in the control group was found, whereas the treatment group had a slight increase of $0.04 \mathrm{~mm}$. Intermolar width increased by $1.5 \mathrm{~mm}$ in the treatment group, as compared with only $0.4 \mathrm{~mm}$ in the control group. Inter-
Table 1. Changes in mandibular molar and incisor position during the study period in the treatment and control group recorded by lateral cephalometric X-ray

\begin{tabular}{lcll}
\hline & $\begin{array}{l}\text { Treatment group } \\
\text { (T0-T1) }\end{array}$ & $\begin{array}{l}\text { Control group } \\
\text { (T0-T1) }\end{array}$ & $\mathrm{p}$ \\
\hline 6T0-6T1, mm & $+0.6 \pm 0.8$ & $+1.9 \pm 0.9$ & $<0.05$ \\
6(Cr)T0-6(Cr)T1, mm angular, $^{\circ}$ & $+0.3 \pm 0.2$ & $+1.3 \pm 0.6$ & $<0.05$ \\
1T0-1T1, mm & $-0.06 \pm 0.8$ & $+1.9 \pm 0.8$ & $<0.05$ \\
1(Cr)T0-1(Cr)T1, mm & $+0.5 \pm 0.9$ & $-0.4 \pm 0.7$ & $<0.05$ \\
1 angular, ${ }^{\circ}$ & $+0.3 \pm 0.5$ & $-0.2 \pm 0.4$ & $<0.05$ \\
& $+0.7 \pm 0.8$ & $-2.1 \pm 0.9$ & $<0.01$ \\
\hline
\end{tabular}

Values denote means \pm SD unless specified otherwise.

Table 2. Change in arch dimension during the study period as measured from study models in the treatment and control group

\begin{tabular}{lcll}
\hline & $\begin{array}{c}\text { Treatment group } \\
\text { (T0-T1), mm }\end{array}$ & $\begin{array}{l}\text { Control group } \\
\text { (T0-T1), mm }\end{array}$ & \\
\hline Arch length (6-1) & $+0.04 \pm 0.2$ & $-1.8 \pm 0.7$ & $<0.01$ \\
Intermolar width & $+1.5 \pm 0.9$ & $+0.4 \pm 0.6$ & $<0.05$ \\
Intercanine width & $+1.2 \pm 0.8$ & $+0.2 \pm 0.7$ & $<0.05$ \\
\hline
\end{tabular}

Values denote means \pm SD unless specified otherwise.

canine width increased by $1.2 \mathrm{~mm}$ in the treatment group, as compared with only $0.2 \mathrm{~mm}$ in the control group.

The mean changes and standard deviations for the 3 groups are displayed in table 3 . In the MPR group, the mesiobuccal cusp drifted mesially by $0.9 \pm 0.2 \mathrm{~mm}$, whereas the MSD and MAR mesial drift values were $0.5 \pm 0.2 \mathrm{~mm}$ and $0.4 \pm 0.1 \mathrm{~mm}$, respectively. The molar Cr in the MPR group moved forward by $0.6 \pm 0.2 \mathrm{~mm}$, while the MSD and MAR Cr moved forward by $0.3 \pm 0.1 \mathrm{~mm}$ and $0.2 \pm$ $0.1 \mathrm{~mm}$, respectively. The molar tipped backward by -0.4 $\pm 0.5^{\circ}$ in the MSD group and by $-0.3 \pm 0.7^{\circ}$ in the MAR group; instead, in the MPR group, the molar tipped forward by $0.5 \pm 0.6^{\circ}$. The incisal edge advanced by $0.8 \pm$ $0.2 \mathrm{~mm}$ in the MPR group, and by $0.3 \pm 0.1 \mathrm{~mm}$ and 0.2 $\pm 0.1 \mathrm{~mm}$ in the MSD group and MAR group, respectively. In the MPR group, the molar Cr advanced by $0.1 \pm$ $0.5 \mathrm{~mm}$, in the MSD group it advanced by $0.4 \pm 0.4 \mathrm{~mm}$, and in the MAR group by $0.5 \pm 0.3 \mathrm{~mm}$. The incisor tipped forward by $0.7 \pm 0.8^{\circ}, 0.3 \pm 0.7^{\circ}$ and $0.9 \pm 0.8^{\circ}$ in the MSD, MPR and MAR groups, respectively.

The differences were all found to be statistically significant $(\mathrm{p}<0.05)$ between the MAR group and the other 2 groups (MSD and MAR). No significant differences were found between the MSD group and the MAR group. 
Table 3. Changes in mandibular molar and incisor position during the study period in the MPR, MSD and MAR groups, recorded by lateral cephalometric X-ray

\begin{tabular}{|c|c|c|c|c|c|c|}
\hline & \multirow[t]{2}{*}{ MSD group } & \multirow[t]{2}{*}{ MPR group } & \multirow[t]{2}{*}{ MAR group } & \multicolumn{3}{|l|}{$\mathrm{p}$} \\
\hline & & & & MSD-MPR & MSD-MAR & MPR-MAR \\
\hline 6T0-6T1, mm & $+0.5 \pm 0.2$ & $+0.9 \pm 0.2$ & $+0.4 \pm 0.1$ & $<0.05$ & NS & $<0.05$ \\
\hline $6(\mathrm{Cr}) \mathrm{T} 0-6(\mathrm{Cr}) \mathrm{T} 1, \mathrm{~mm}$ & $+0.3 \pm 0.1$ & $+0.6 \pm 0.2$ & $+0.2 \pm 0.1$ & $<0.05$ & NS & $<0.05$ \\
\hline 6 angular, $^{\circ}$ & $-0.4 \pm 0.5$ & $+0.5 \pm 0.6$ & $-0.3 \pm 0.7$ & $<0.05$ & NS & $<0.01$ \\
\hline $1 \mathrm{~T} 0-1 \mathrm{~T} 1, \mathrm{~mm}$ & $+0.3 \pm 0.1$ & $+0.8 \pm 0.2$ & $+0.2 \pm 0.1$ & $<0.05$ & NS & $<0.05$ \\
\hline $1(\mathrm{Cr}) \mathrm{T} 0-1(\mathrm{Cr}) \mathrm{T} 1, \mathrm{~mm}$ & $+0.4 \pm 0.4$ & $+0.1 \pm 0.5$ & $+0.5 \pm 0.3$ & $<0.05$ & NS & $<0.05$ \\
\hline 1 angular, $^{\circ}$ & $+0.7 \pm 0.8$ & $+0.3 \pm 0.7$ & $+0.9 \pm 0.8$ & $<0.05$ & NS & $<0.05$ \\
\hline
\end{tabular}

Values denote means \pm SD unless specified otherwise. NS = Not significant.

Table 4. Change in arch dimension during the study period as measured by study models in the MPR, MSD and MAR groups

\begin{tabular}{|c|c|c|c|c|c|c|}
\hline & \multirow{2}{*}{$\begin{array}{l}\text { MSD group, } \\
\mathrm{mm}\end{array}$} & \multirow{2}{*}{$\begin{array}{l}\text { MPR group, } \\
\mathrm{mm}\end{array}$} & \multirow{2}{*}{$\begin{array}{l}\text { MAR group, } \\
\mathrm{mm}\end{array}$} & \multicolumn{3}{|l|}{$\underline{\mathrm{p}}$} \\
\hline & & & & MSD-MPR & MSD-MAR & MPR-MAR \\
\hline Arch length (6-1) & $+0.05 \pm 0.2$ & $+0.02 \pm 0.1$ & $+0.07 \pm 0.1$ & NS & NS & NS \\
\hline Intermolar width & $+1.7 \pm 0.8$ & $+0.9 \pm 0.7$ & $+2.0 \pm 0.9$ & $<0.05$ & NS & $<0.05$ \\
\hline Intercanine width & $+1.3 \pm 0.9$ & $+0.7 \pm 0.8$ & $+1.6 \pm 0.6$ & $<0.05$ & NS & $<0.05$ \\
\hline
\end{tabular}

Values denote means \pm SD unless specified otherwise. NS $=$ Not significant.

The mean changes and standard deviations of the arch length, intermolar width and intercanine width in the 3 groups are given in table 4 . The change in arch length was not statistically significant between the 3 groups, but the intermolar and intercanine width changes were all found to be statistically significant $(\mathrm{p}<0.05)$ between the MPR group and the other 2 groups (MSD and MAR). No significant differences were found between the MSD and MAR groups.

\section{Discussion}

Shortening of the dental arch, in the transition from mixed to permanent dentition, is well established, and a lingual arch is commonly used as a holding device to maintain mandibular arch length. Findings from previous studies [5, 16-18] demonstrated that a passive lingual arch is effective in preserving a lower arch length during the transition from mixed to permanent dentition.

In this study we found that the arch length increased by $0.04 \mathrm{~mm}$ in patients treated with lingual arches. This observation is similar to the findings by Singer [10], who reported an increase of $0.2 \mathrm{~mm}$, while another author noted a slight decrease in arch length. De Baets and Chiarini [19] found a decrease of $0.5 \mathrm{~mm}$ that they attributed to lingual tipping of the incisor, and Rebellato et al. [5] reported a decrease of $0.07 \mathrm{~mm}$. Brennan and Gianelly [11] found a decrease of $0.44 \mathrm{~mm}$, but they also noted a large variability in the effects of the lingual arch; in fact the arch length decreased by an average of $-0.44 \pm$ $1.35 \mathrm{~mm}$, with a range from -4.79 to $+2.9 \mathrm{~mm}$. They understood that the changes seen in incisor and molar positions during the treatment with lingual arches were influenced by facial growth. Also, the study by Germane et al. [20] demonstrated that the change in arch perimeter was determined by different factors such as tooth angulation, arch width and length, and the curve of Spee, and that these factor are influenced by facial growth.

The intercanine width increased by $1.2 \mathrm{~mm}$ in the treated group and $0.2 \mathrm{~mm}$ in the control group, similar to the increase reported by Brennan and Gianelly [11], who noted a 1.49-mm increase in intercanine width after lingual arch therapy, and this increment was attributed to the developmental changes in arch dimension. De Baets and Chiarini [19] found that the intercanine dimension increased by 11 $\mathrm{mm}$ after lingual arch therapy, which they attributed to the lateral migration of the canines as they drifted into the 
leeway space. However, Bishara et al. [21] and Singer [10] found only an $0.5-\mathrm{mm}$ increase in intercanine width after lingual arch placement. The differences in intercanine width increase could have been due to distolateral migration of the canines as they moved into the leeway space, as noted by De Baets and Chiarini [19], or due to different mandibular growth patterns. The findings obtained in this investigation demonstrate that the patients with an MPR exhibited a smaller increase in intercanine width $(0.7$ $\mathrm{mm}$ ) compared to the other two groups.

However, no study has ever reported the difference between subjects with MPR and MAR. This study was designed to evaluate whether or not the placement of a lingual arch had the same effects in subjects with different mandibular growth patterns. The findings obtained in this investigation demonstrate that the patients with an MPR exhibited a greater mesial movement of the first molars compared to the other two groups. However, in subjects with MPR, the arch length did not decrease due to a greater mandibular incisor advancement. Therefore, the null hypothesis had to be rejected. Mandibular morphology is very important during the treatment with lingual arches, in fact the stability of the molar is influenced by the subject's natural muscular anchorage. In the dolichofacial subject, the muscle structure is weaker and is easily overpowered by the forces; in fact, the molar moves mesially and the incisor moves forward without any blocking by the lips. In the mesiofacial and brachyfacial subjects, the strongest muscle allows a greater stability of the molars and incisors, keeping the effect of the lingual arch stable.

The results from this study show that mandibular lingual arches can help to reduce the arch perimeter loss that occurs during the transition from mixed to permanent dentition. Therefore, the subject's mandibular morphology should always be considered before treatment and, in subjects with MPR, there is no effective reduction in the molar mesial migration. However, there is a mandibular incisor advancement and tipping which may, or may not, be desirable depending on ultimate treatment goals.

\section{Conclusion}

The results of the study suggest that the lingual arch is good appliance for preserving arch length, but the effect of this is influenced by mandibular growth patterns; in fact, in patients with MPR, the lingual arch preserves arch length but with mandibular incisor advancement and tipping. Hence, it is very important to consider mandibular growth patterns before lingual arch treatment.

\section{References}

1 Villalobos F, Sinha P, Nanda R: Longitudinal assessment of vertical and sagittal control in the mandibular arch by the mandibular fixed lingual arch. Am J Orthod Dentofacial Orthop 2000;118:366-370.

2 Nance HN: The limitations of orthodontic treatment. 1. Mixed dentition diagnosis and treatment. Am J Orthod Oral Surg 1947;33: 177-223.

3 Moyers RE, van der Linden FPGM, Riolo ML, McNamara JA Jr: Standards of Human Occlusal Development. Monograph 5. Craniofacial Growth Series. Ann Arbor, University of Michigan, 1976.

4 Moorrees CFA, Chada JM: Available space for incisors during dental development: a growth study based on physiologic age. Angle Orthod 1965;35:12-22.

-5 Rebellato J, Lindauer S, Rubenstein L, Isaacson R, Davidovich M, Vroom K: Lower arch perimeter preservation using the lingual arch. Am J Orthod Dentofacial Orthop 1997; 112:449-456.

6 Owen DG: The incidence and nature of space closure following the premature extraction of deciduous teeth: a literature survey. Am J Orthod Dentofacial Orthop 1971;59:37-49.
7 Wright GZ, Kennedy DB: Space control in the primary and mixed dentition. Dent Clin North Am 1978;22:579-601.

8 Eastwood AW: The lingual arch in space control. Dent Clin North Am 1968;Jul:383-397.

$\checkmark 9$ Odom WM: Mixed dentition treatment with cervical traction and lower lingual arch. Angle Orthod 1983;53:329-342.

10 Singer J: The effect of the passive lingual archwire on the lower denture. Angle Orthod 1974;44:146-155.

11 Brennan M, Gianelly A: The use of the lingual arch in the mixed dentition to resolve incisor crowding. Am J Orthod Dentofacial Orthop 2000;117:81-85.

12 Björk A: Variations in the growth pattern of the human mandible: longitudinal radiographic study by the implant method. J Dent Res 1963;42:400-411.

13 Björk A: Prediction of mandibular growth rotation. Am J Orthod 1969;55:585-589.

14 Ricketts RM: The influence of orthodontic treatment on facial growth and development. Angle Orthod 1960;30:103-133.
15 Jarabak JR, Fizzel JA: Technique and Treatment with Light-Wire Edgewise Appliances, ed 2. St Louis, Mosby, 1972.

16 Dugoni S, Lee JS, Valera J, Dugoni A: Early mixed dentition treatment: postretention evaluation of stability and relapse. Angle Orthod 1995;65:311-319.

17 Gianelly A: Leeway space and the resolution of crowding in the mixed dentition. Semin Orthod 1995;1:188-194.

18 Miotti F: The passive lingual arch in first bicuspid extraction. Angle Orthod 1984;54: 163-175.

19 De Baets J, Chiarini M: The pseudo-Class I: a newly defined type of malocclusion. J Clin Orthod 1995;29:73-88.

20 Germane N, Lindauer SJ, Rubenstein LK, Revere JH, Isaacson RJ: Increase in arch perimeter due to orthodontic expansion. Am J Orthod Dentofacial Orthop 1991;100:421-427.

21 Bishara SE, Jakobsen JR, Treder J, Nowak A: Arch width changes from 6 weeks to 45 years of age. Am J Orthod Dentofacial Orthop 1997;111:401-409. 